\title{
On the Chermak-Delgado lattice of a finite group
}

\author{
Ryan McCulloch and Marius Tărnăuceanu
}

May 30, 2019

\begin{abstract}
By imposing conditions upon the index of a self-centralizing subgroup of a group, and upon the index of the center of the group, we are able to classify the Chermak-Delgado lattice of the group. This is our main result. We use this result to classify the Chermak-Delgado lattices of dicyclic groups and of metabelian $p$-groups of maximal class.
\end{abstract}

MSC2000 : Primary 20D30; Secondary 20D60, 20 D99.

Key words: Chermak-Delgado measure, Chermak-Delgado lattice, ChermakDelgado subgroup, subgroup lattice, (generalized) dicyclic group, metabelian group.

\section{Introduction}

Throughout this paper, $G$ will denote a finite group. Denote by

$$
m_{G}(H)=|H|\left|C_{G}(H)\right|
$$

the Chermak-Delgado measure of a subgroup $H$ of $G$ and let $m^{*}(G)=\max \left\{m_{G}(H) \mid H \leq G\right\}$ and $\mathcal{C D}(G)=\left\{H \leq G \mid m_{G}(H)=m^{*}(G)\right\}$.

The set $\mathcal{C D}(G)$ forms a modular, self-dual sublattice of the lattice of subgroups of $G$, which is called the Chermak-Delgado lattice of $G$. It was first introduced by Chermak and Delgado [5], and revisited by Isaacs [7]. In the last years there has been a growing interest in understanding this lattice (see e.g. $[1,2,3,4,6,8,9,10,13,14,15,18]$ ). Recall two important properties of the Chermak-Delgado lattice that will be used in our paper: 
- if $H \in \mathcal{C D}(G)$, then $C_{G}(H) \in \mathcal{C D}(G)$ and $C_{G}\left(C_{G}(H)\right)=H$;

- the minimum subgroup $M(G)$ of $\mathcal{C D}(G)$ (called the Chermak-Delgado subgroup of $G$ ) is characteristic, abelian, and contains $Z(G)$.

For a positive integer $n \geq 1$, the dicyclic group of order $4 n$, usually denoted by $D i c_{4 n}$, is defined as

$$
D i c_{4 n}=\left\langle a, x \mid a^{2 n}=1, x^{2}=a^{n}, a^{x}=a^{-1}\right\rangle .
$$

This has the following generalization: given an arbitrary abelian group $A$ of order $2 n$, the generalized dicyclic group induced by $A$ is defined as

$$
\operatorname{Dic}_{4 n}(A)=\left\langle A, x \mid x^{4}=1, x^{2} \in A \backslash\{1\}, a^{x}=a^{-1}, \forall a \in A\right\rangle .
$$

Obviously, we have $D i c_{4 n}\left(\mathbb{Z}_{2 n}\right)=D i c_{4 n}$. It is also easy to see that if $\exp (A)=$ 2 , that is that $A$ is an elementary abelian 2-group, then $D i c_{4 n}(A)$ is abelian and consequently $\mathcal{C D}\left(D i c_{4 n}(A)\right)=\left\{D i c_{4 n}(A)\right\}$. Note that if $\exp (A) \neq 2$, then

$$
Z\left(\operatorname{Dic}_{4 n}(A)\right)=\left\{a \in A \mid a^{2}=1\right\} \cong \frac{A}{A^{2}},
$$

where $A^{2}=\left\{a^{2} \mid a \in A\right\}$.

A finite group $G$ is said to be metabelian if the derived subgroup, $G^{\prime}$, is abelian. Equivalently, a finite group $G$ is metabelian if there exists an abelian normal subgroup $A$ of $G$ so that $G / A$ is abelian.

A finite $p$-group $G$ of order $p^{n}$ is said to be of maximal class if the nilpotence class of $G$ is $n-1$. The following results on $p$-groups will be useful to us. Lemma 1.1 appears in (4.26), [12], II, Lemma 1.2 appears in Theorem 2.4 of [16], and Lemma 1.3 at end of [14].

Lemma 1.1. Any group of order $p^{4}$ contains an abelian subgroup of order $p^{3}$.

Lemma 1.2. Suppose $G$ is a p-group of order $p^{n}$ and $G$ is of maximal class. Then $|Z(G)|=p,\left|G: G^{\prime}\right|=p^{2}$, and for each $2 \leq i \leq n$, we have that $G_{i}$ is the unique normal subgroup of $G$ order $p^{n-i}$, where $G_{i}$ is the ith term in the lower central series for $G$.

Lemma 1.3. Let $G$ be a p-group of maximal class and of order $p^{5}$. If $m^{*}(G)=p^{6}$, then $\mathcal{C D}(G)=\left\{G, T, G^{\prime}, A_{1}, \ldots, A_{p}, Z(T), Z(G)\right\}$, where $|T|=$ $p^{4},\left|G^{\prime}\right|=\left|A_{i}\right|=p^{3}$ for each $i,|Z(T)|=p^{2}$, and $G^{\prime}, A_{1}, \ldots, A_{p}$ are all abelian and distinct. Also, none of $A_{1}, \ldots, A_{p}$ are normal in $G$. 


\section{Main Results}

In this section we present a result which generalizes Proposition 7 in [10]. It will be used both in Sections 3 and 4. A subgroup $A$ of $G$ is said to be self-centralizing if $C_{G}(A)=A$. An important observation used here is that if $T \in \mathcal{C D}(G)$, then since $Z(G) \leq T$, we have that $|G: T|$ divides $|G: Z(G)|$. So by imposing conditions on $|G: Z(G)|$, we are able to obtain results about $\mathcal{C D}(G)$.

Theorem 2.1. Let $G$ be a finite group, let $p$ be a prime, and among the self-centralizing subgroups of $G$, let $A$ be one of maximum order.

1) If $G$ is abelian, then $\mathcal{C D}(G)=\{G\}$.

2) If $|G: Z(G)|=p^{2}$, then $\mathcal{C D}(G)=\left\{Z(G), A, A_{1}, \ldots, A_{p}, G\right\}$ is a quasiantichain of width $p+1$, with each $A_{i}$ abelian.

3) If $|G: A|=p$ and $|G: Z(G)|=p^{i}$ with $i>2$, then $\mathcal{C D}(G)=\{A\}$.

If $p$ is the smallest prime divisor of $|G|$ and $|G: A|=p$ and $\mid G$ : $Z(G) \mid>p^{2}$, then $\mathcal{C D}(G)=\{A\}$.

4) Suppose $|G: A|=p^{2}$.

a) If $|G: Z(G)|=p^{3}$, then $\mathcal{C D}(G)=\{Z(G), G\}$.

If $p$ is the smallest prime divisor of $|G|$ and $p^{2}<|G: Z(G)|<p^{4}$, then $\mathcal{C D}(G)=\{Z(G), G\}$.

b) If $|G: Z(G)|=p^{4}$, then

$\mathcal{C D}(G)=\left\{Z(G), Z\left(T_{1}\right), \ldots, Z\left(T_{n}\right), A, A_{1}, \ldots, A_{m}, T_{1}, \ldots, T_{n}, G\right\}$

where $T_{1}, \ldots, T_{n}(n \geq 0)$ are all of the subgroups of index $p$ in $G$ with centers that have index $p^{3}$ in $G$, and $A_{1}, \ldots, A_{m}(m \geq 0)$ are all of the subgroups (other than $A$ ) of index $p^{2}$ in $G$ with centralizers that have index $p^{2}$ in $G$. Furthermore, if $n \geq 1$, then $m \geq p$.

c) If $|G: Z(G)|=p^{i}$ with $i>4$ and if $G$ possesses a subgroup, $T$, of index $p$ in $G$ with center that has index $p^{3}$ in $G$, then $\mathcal{C D}(G)=$ $\left\{Z(T), A, A_{1}, \ldots, A_{p}, T\right\}$ is a quasi-antichain of width $p+1$, with each $A_{i}$ abelian. 
If $p$ is the smallest prime divisor of $|G|$ and if $|G: Z(G)|>p^{4}$ and if $G$ possesses a subgroup, $T$, of index $p$ in $G$ with center that has index $p^{3}$ in $G$, then $\mathcal{C D}(G)=\left\{Z(T), A, A_{1}, \ldots, A_{p}, T\right\}$ is a quasi-antichain of width $p+1$, with each $A_{i}$ abelian.

d) If $|G: Z(G)|=p^{i}$ with $i>4$ and if $G$ does not possess a subgroup, $T$, of index $p$ in $G$ with center that has index $p^{3}$ in $G$, then $\mathcal{C D}(G)=\{A\}$

If $p$ is the smallest prime divisor of $|G|$ and if $|G: Z(G)|>p^{4}$ and if $G$ does not possess a subgroup, $T$, of index $p$ in $G$ with center that has index $p^{3}$ in $G$, then $\mathcal{C D}(G)=\{A\}$.

Proof. Item 1 is clear.

To see item 2, note first that $|G: G||G: Z(G)|=|G: Z(G)|=p^{2} \leq \mid G$ : $T \| G: C_{G}(T) \mid$ for any subgroup $T$ of $G$ with $Z(G) \leq T$, and so $G, Z(G) \in$ $\mathcal{C D}(G)$. Note that $G / Z(G) \cong C_{p} \times C_{p}$, and so there are exactly $p+1$ subgroups $H$ with $Z(G)<H<G$. For any such $H$, we have that $H=$ $\langle Z(G), x\rangle$ for some $x \in H \backslash Z(G)$, and thus $H$ is abelian. It follows that $\mathcal{C D}(G)=\left\{Z(G), A, A_{1}, \ldots, A_{p}, G\right\}$ is a quasi-antichain of width $p+1$, with each $A_{i}$ abelian.

We prove both parts of item 3 simultaneously. Note that $|G: G| \mid G$ : $Z(G)|=| G: Z(G)\left|>p^{2}=\right| G: A|| G: A \mid$, and so $G, Z(G) \notin \mathcal{C D}(G)$. Note that $|G: A||G: A|=p^{2} \leq|G: T|\left|G: C_{G}(T)\right|$ for any subgroup $T$ of $G$ with $Z(G) \leq T$, and so $A \in \mathcal{C D}(G)$. Now $A$ is a maximal subgroup of $G$ and $G \notin \mathcal{C D}(G)$, and so $A$ is the largest member of $\mathcal{C D}(G)$. But $A$ is self-centralizing and therefore $A$ is also the least member of $\mathcal{C D}(G)$. Hence $\mathcal{C D}(G)=\{A\}$.

We prove both parts of item 4.a simultaneously. Note that $\mid G: A \| G$ : $A\left|=p^{4}>\right| G: Z(G)|=| G: G|| G: Z(G) \mid$, and so $A \notin \mathcal{C D}(G)$. Since among the self-centralizing subgroups of $G, A$ is one of maximum order, it follows that $\mathcal{C D}(G)$ does not contain any self-centralizing subgroups. Among the abelian subgroups of $\mathcal{C D}(G)$, let $T$ be a maximal one. If $T \neq Z(G)$, then we have that $T<C_{G}(T)<G$, and furthermore we have that $\left|C_{G}(T): T\right|$ cannot be a prime. This is true because if $\left|C_{G}(T): T\right|$ is a prime, then $C_{G}(T) / T$ is cyclic, and hence $C_{G}(T)$ is abelian, and so $T$ would not be maximal among the abelian subgroups in $\mathcal{C D}(G)$. It follows that $\mid G: T \| G$ : $C_{G}(T)\left|\geq p^{4}>\right| G: G|| G: Z(G) \mid$, a contradiction. Hence $T=Z(G)$ is the only abelian subgroup in $\mathcal{C D}(G)$. If $H \in \mathcal{C D}(G)$ is a subgroup of index $p$ in 
$G$ with $C_{G}(H)$ a subgroup of index $p^{2}$ in $G$, then $\left|C_{G}(H): Z(G)\right|<p^{2}$, and hence is prime. But then $C_{G}(H)$ is abelian, a contradiction. It follows that $\mathcal{C D}(G)=\{Z(G), G\}$.

To see item 4.b, note that $p^{4}=|G: G||G: Z(G)|=|G: A||G: A|$. Since among the self-centralizing subgroups of $G, A$ is one of maximum order, we have that any other self-centralizing subgroup $A^{\prime}$ of $\mathcal{C D}(G)$ will have that $p^{4}=\left|G: A^{\prime}\right|\left|G: A^{\prime}\right|$. If $T$ is an abelian subgroup of $\mathcal{C D}(G)$ with $T<C_{G}(T)<G$ and $C_{G}(T)$ nonabelian, then as we saw in the proof of item 4.a, we have that $|G: T|\left|G: C_{G}(T)\right| \geq p^{4}$. It follows then that $G, Z(G), A \in \mathcal{C D}(G)$. And hence

$$
\left\{Z(G), Z\left(T_{1}\right), \ldots, Z\left(T_{n}\right), A, A_{1}, \ldots, A_{m}, T_{1}, \ldots, T_{n}, G\right\} \subseteq \mathcal{C D}(G)
$$

where $T_{1}, \ldots, T_{n}(n \geq 0)$ are all of the subgroups of index $p$ in $G$ with centers that have index $p^{3}$ in $G$, and $A_{1}, \ldots, A_{m}(m \geq 0)$ are all of the subgroups (other than $A$ ) of index $p^{2}$ in $G$ with centralizers that have index $p^{2}$ in $G$.

We also have that

$$
\mathcal{C D}(G) \subseteq\left\{Z(G), Z\left(T_{1}\right), \ldots, Z\left(T_{n}\right), A, A_{1}, \ldots, A_{m}, T_{1}, \ldots, T_{n}, G\right\} .
$$

This is true because, otherwise, if some $H \in \mathcal{C D}(G)$ has that $|G: H|=p$ and $\left|G: C_{G}(H)\right|=p^{3}$, but $Z(H)<C_{G}(H)$, then since $Z(H)=H \cap C_{G}(H) \in$ $\mathcal{C D}(G)$, it would follow that $Z(H)=Z(G)$ and thus $H=G$, a contradiction.

If $n \geq 1$, then for some $T \in\left\{T_{1}, \ldots, T_{n}\right\}$, we have that $Z(T)<A<T$ in $\mathcal{C D}(G)$. This is true because, otherwise, we would have $Z(G)<A<G$ and $Z(G)<Z(T)<T<G$ as maximal chains in $\mathcal{C D}(G)$, contradicting the modularity of $\mathcal{C D}(G)$. We can apply item 2 in Theorem 2.1 to the group $T$, to conclude that there are $p$ additional self-centralizing groups in $\mathcal{C D}(T)$, and since all of these are self-centralizing, they are also all in $\mathcal{C D}(G)$.

We prove both parts of item 4.c simultaneously. Note that $|G: G| \mid G$ : $Z(G)\left|>p^{4}=\right| G: A|| G: A \mid$, and so $G, Z(G) \notin \mathcal{C D}(G)$. As we saw before, if $S$ is an abelian subgroup of $\mathcal{C D}(G)$ with $S<C_{G}(S)<G$ and $C_{G}(S)$ nonabelian, then $|G: S|\left|G: C_{G}(S)\right| \geq p^{4}$. It follows that $A, T, Z(T) \in$ $\mathcal{C D}(G)$, and since $T$ is maximal in $G$, we have that $T$ is the largest member of $\mathcal{C D}(G)$. And so applying Theorem 2.1 item 2 to the group $T$, we obtain that $\mathcal{C D}(G)=\left\{Z(T), A, A_{1}, \ldots, A_{p}, T\right\}$ is a quasi-antichain of width $p+1$, with each $A_{i}$ abelian.

We prove both parts of item 4.d simultaneously. As we saw in the proof of item 4.c, $G, Z(G) \notin \mathcal{C D}(G)$ and $A \in \mathcal{C D}(G)$. But since there is no subgroup 
of index $p$ in $\mathcal{C D}(G)$, we have that $A$ is the largest member of $\mathcal{C D}(G)$, and since $A$ is self-centralizing, $A=C_{G}(A)$ is the least member of $\mathcal{C D}(G)$, and we conclude that $\mathcal{C D}(G)=\{A\}$.

Items 3, 4.c, and 4.d in Theorem 2.1 have interesting consequences due to the uniqueness of the largest member of the Chermak-Delgado lattice. We collect them below as a corollary.

Corollary 2.2. Let $G$ be a finite group, let $p$ be a prime, and among the self-centralizing subgroups of $G$, let $A$ be one of maximum order.

1) If $|G: A|=p$ and $|G: Z(G)|=p^{i}$ with $i>2$, then $A$ is unique.

If $p$ is the smallest prime divisor of $|G|$ and $|G: A|=p$ and $\mid G$ : $Z(G) \mid>p^{2}$, then $A$ is unique.

2) If $|G: A|=p^{2}$ and $|G: Z(G)|=p^{i}$ with $i>4$ and if $G$ possesses $a$ subgroup, $T$, of index $p$ in $G$ with center that has index $p^{3}$ in $G$, then $T$ is unique.

If $p$ is the smallest prime divisor of $|G|$ and if $|G: A|=p^{2}$ and $\mid G$ : $Z(G) \mid>p^{4}$ and if $G$ possesses a subgroup, $T$, of index $p$ in $G$ with center that has index $p^{3}$ in $G$, then $T$ is unique.

3) If $|G: A|=p^{2}$ and $|G: Z(G)|=p^{i}$ with $i>4$ and if $G$ does not possess a subgroup, $T$, of index $p$ in $G$ with center that has index $p^{3}$ in $G$, then $A$ is unique.

If $p$ is the smallest prime divisor of $|G|$ and if $|G: A|=p^{2}$ and $\mid G$ : $Z(G) \mid>p^{4}$ and if $G$ does not possess a subgroup, $T$, of index $p$ in $G$ with center that has index $p^{3}$ in $G$, then $A$ is unique.

Examples for items 4.a and 4.b in Theorem 2.1 that are chains of length 1 and length 2 (and so in the case of item 4.b, $n=0$ and $m=0$ ), can be found in [3], see Corollary 2.2, Proposition 2.3, Corollary 2.5, and Proposition 2.6 there.

An example for item 4.b in Theorem 2.1 with $n=1$ and $m=p$ will be given in Section 4.

Another example for item 4.b in Theorem 2.1 is found by taking $G$ to be one of the two extraspecial $p$-groups of order $p^{5}$. $|Z(G)|=p$ (i.e. $\mid G$ : $\left.Z(G) \mid=p^{4}\right)$ and $G$ possesses a maximal self-centralizing elementary abelian subgroup of order $p^{3}$ (i.e. of index $p^{2}$ in $G$ ). The Chermak-Delgado lattice 
of extraspecial p-groups is known, see Example 2.8 in [6]. And so we have that $\mathcal{C D}(G)$ is isomorphic to the subgroup lattice of an elementary abelian $p$-group of order $p^{4}$. Using the notation of item 4.b in Theorem 2.1, we have that $n=p^{3}+p^{2}+p+1$ and $m=\left(p^{2}+1\right)\left(p^{2}+p+1\right)-1$.

Further examples of groups and CD lattices arising from Theorem 2.1 will be explored in the next sections.

\section{$3 \quad$ Dicyclic groups}

As a consequence of items 2 and 3 in Theorem 2.1, we classify the ChermakDelgado lattice of $\operatorname{Dic}_{4 n}(A)$, where $A$ is an abelian group of order $2 n$.

Note that $\mathcal{C D}\left(\operatorname{Dic}_{4 n}(A)\right)=\{A\}$ if and only if $\left|\operatorname{Dic}_{4 n}(A): Z\left(D i c_{4 n}(A)\right)\right|>$ 4, which is equivalent to $\left|A^{2}\right|>2$. If $A$ is not an elementary abelian 2-group, this means that $A$ is not a direct product of an elementary abelian 2-group and $\mathbb{Z}_{4}$.

Theorem 3.1. Let $A$ be an abelian group of order $2 n$ and $\operatorname{Dic}_{4 n}(A)$ be the generalized dicyclic group induced by $A$. Then we have:

a) If $A$ is not of type $\mathbb{Z}_{2}^{m} \times \mathbb{Z}_{4}$ with $m \in \mathbb{N}$, then $\mathcal{C D}\left(\operatorname{Dic}_{4 n}(A)\right)$ is a chain of length 0 , namely $\mathcal{C D}\left(\operatorname{Dic}_{4 n}(A)\right)=\left\{\operatorname{Dic}_{4 n}(A)\right\}$ for $\exp (A)=2$, and $\mathcal{C D}\left(\operatorname{Dic}_{4 n}(A)\right)=\{A\}$ for $\exp (A) \neq 2$.

b) If $A$ is of type $\mathbb{Z}_{2}^{m} \times \mathbb{Z}_{4}$ with $m \in \mathbb{N}$, then $\mathcal{C D}\left(\operatorname{Dic}_{4 n}(A)\right)$ is a quasiantichain of width 3 , namely the lattice interval between $Z\left(\operatorname{Dic}_{4 n}(A)\right)$ and $\operatorname{Dic}_{4 n}(A)$.

As a corollary, we describe the Chermak-Delgado lattice of $D i c_{4 n}$.

Corollary 3.2. Under the previous notation, we have:

a) If $n \neq 2$, then $\mathcal{C D}\left(\right.$ Dic $\left._{4 n}\right)$ is a chain of length 0 , namely $\mathcal{C D}\left(\right.$ Dic $\left._{4 n}\right)=$ $\left\{D_{i c_{4 n}}\right\}$ for $n=1$, and $\mathcal{C D}\left(D i c_{4 n}\right)=\{\langle a\rangle\}$ for $n \geq 3$.

b) If $n=2$, then $\mathcal{C D}\left(\right.$ Dic $\left._{4 n}\right)$ is a quasi-antichain of width 3, namely the lattice interval between $Z\left(D i c_{4 n}\right)$ and Dic $_{4 n}$.

The following appears in [10]. 
Theorem 3.3. Let $G$ be a finite group which can be written as $G=A B$, where $A$ and $B$ are abelian subgroups of relatively prime orders and $A$ is normal. Then

$$
m(G)=|A|^{2}\left|C_{B}(A)\right|^{2} \text { and } \mathcal{C D}(G)=\left\{A C_{B}(A)\right\} .
$$

Corollary 3.4. Suppose $A$ is a finite abelian group. There exists a nonabelian finite group $G$ so that $\mathcal{C D}(G)=\{A\}$ if and only if $A \neq 1, A \nsubseteq \mathbb{Z}_{2}$, $A \not \mathbb{Z}_{4}$, and $A \not \mathbb{Z}_{2} \times \mathbb{Z}_{4}$.

Proof. $\rightarrow$ Suppose $\mathcal{C D}(G)=\{A\}$ and $G$ is non-abelian. If $A=1$, then $G=1$ is abelian, a contradiction. Since $A$ is self-centralizing and normal in $G$, we have that $G / A$ embeds into $A$ ut $(A)$. If $A \cong \mathbb{Z}_{2}$, then $G / A$ embeds into $\operatorname{Aut}(A)=1$, which contradicts $G$ being non-abelian. If $A \cong \mathbb{Z}_{4}$, then $G / A$ embeds into $A u t(A)$ which has order 2 , and so $|G|=8$ and $C D(G)$ would not be a chain of length zero by item 2 in Theorem 2.1. Suppose $A \cong \mathbb{Z}_{2} \times \mathbb{Z}_{4}$. Then $G / A$ embeds into $A u t(A)$ which is a dihedral group of size 8 . And so $G$ is a 2-group. Now, $m^{*}(G)=64$, and since $G \notin \mathcal{C D}(G)$ and $G$ has a nontrivial center, it follows that $|G|=16$ and $|Z(G)|=2$. Note, however, that given $x \in \operatorname{Aut}(A)$ of order $2, x$ must fix at least four elements of $A$. This is due to the structure of $\operatorname{Aut}(A)$ when $A \cong \mathbb{Z}_{2} \times \mathbb{Z}_{4}$. And so $|Z(G)|>2$, a contradiction.

$\leftarrow$ If $|A|$ is odd, then let $G=A \rtimes\langle x\rangle$ where $x$ inverts each element of $A$. Then by item 3 in Theorem $2.1, \mathcal{C D}(G)=\{A\}$. If $|A|$ is even, and $A$ is not of type $\mathbb{Z}_{2}^{m} \times \mathbb{Z}_{4}$ with $m \geq 0$, and $\exp (A) \neq 2$, then $G=D i c_{4 n}(A)$ has that $\mathcal{C D}(G)=\{A\}$ by Theorem 3.1. If $A \cong \mathbb{Z}_{2}^{m}$ with $m \geq 2$ or $A \cong \mathbb{Z}_{2}^{m} \times \mathbb{Z}_{4}$ with $m \geq 2$, then there exists $x \in A u t(A)$ of order 3. Let $G=A \rtimes\langle x\rangle$. One can apply Theorem 3.3 to get that $\mathcal{C D}(G)=\{A\}$.

Finally, we present a proposition also applicable to describing $\mathcal{C D}\left(\operatorname{Dic}_{4 n}\right)$.

Proposition 3.5. Let $G$ be a finite group. If $G=H Z(G)$ for some subgroup $H$ of $G$, then $\mathcal{C D}(G)$ and $\mathcal{C D}(H)$ are lattice isomorphic with $\mathcal{C D}(G)=$ $\{X Z(G) \mid X \in \mathcal{C D}(H)\}$.

Proof. Since $G=H Z(G)$, we have that $G / Z(G) \cong H /(Z(G) \cap H)=$ $H / Z(H)$ since $Z(H) \leq Z(G)$. This natural isomorphism between $G / Z(G)$ and $H / Z(H)$ induces a lattice isomorphism between the subgroup intervals $[G: Z(G)]$ and $[H: Z(H)]$, and this lattice isomorphism restricts to a lattice isomorphism between $\mathcal{C D}(G)$ and $\mathcal{C D}(H)$. The result follows. 


\section{Metabelian $p$-groups of maximal class}

By applying Lemmas 1.1, 1.2, 1.3 and Theorem 2.1, we obtain a classification of the Chermak-Delgado lattices of a metabelian $p$-groups of maximal class.

Theorem 4.1. Suppose $G$ is a metabelian p-group of order $p^{n}$ and of maximal class.

1 ) If $n=3$, then $\mathcal{C D}(G)=\left\{Z(G), A, A_{1}, \ldots, A_{p}, G\right\}$ is a quasi-antichain of width $p+1$, with each $A_{i}$ abelian.

2 ) If $G$ possesses an abelian subgroup $A$ so that $|G: A|=p$ and $n>3$, then $\mathcal{C D}(G)=\{A\}$.

3 ) Suppose that $G$ does not possess an abelian subgroup $A$ so that $\mid G$ : $A \mid=p$. Then we have that $n>4$, and, furthermore:

a) If $|G|=p^{5}$, then $\mathcal{C D}(G)=\left\{Z(G), Z(T), G^{\prime}, A_{1}, \ldots, A_{p}, T, G\right\}$, where $|T|=p^{4},\left|G^{\prime}\right|=\left|A_{i}\right|=p^{3}$ for each $i,|Z(T)|=p^{2}$, and $G^{\prime}, A_{1}, \ldots, A_{p}$ are all abelian and distinct. Also, none of $A_{1}, \ldots, A_{p}$ are normal in $G$.

b) If $|G|>p^{5}$ and $G$ possesses a subgroup $T$ of index $p$ so that $|T: Z(T)|=p^{2}$, then $\mathcal{C D}(G)=\left\{Z(T), G^{\prime}, A_{1}, \ldots, A_{p}, T\right\}$, where $|T|=p^{n-1},\left|G^{\prime}\right|=\left|A_{i}\right|=p^{n-2}$ for each $i,|Z(T)|=p^{n-3}$, and $G^{\prime}, A_{1}, \ldots, A_{p}$ are all abelian and distinct. Also, none of $A_{1}, \ldots, A_{p}$ are normal in $G$.

c) If $|G|>p^{5}$ and $G$ does not possess a subgroup $T$ of index $p$ so that $|T: Z(T)|=p^{2}$, then $\mathcal{C D}(G)=\left\{G^{\prime}\right\}$.

Proof. Items 1 and 2 follow directly from items 2 and 3 in Theorem 2.1. The fact that $n>4$ in item 3 follows from Lemma 1.1. Most of Theorem 4.1 part 3 is a straight forward application of Lemma 1.2 and Theorem 2.1 taking $A=G^{\prime}$. In item 3.a, the fact that there is a unique subgroup, $T$, of index $p$ in $G$ in $\mathcal{C D}(G)$ is a nontrivial result that follows from Lemma 1.3 which in turn relies on Lemma 4.5.4 in [14]. In items 3.a and 3.b, the fact that none of $A_{1}, \ldots, A_{p}$ are normal in $G$ follows from Lemma 1.2.

Using Theorem 4.1, we are able to give some necessary and sufficient conditions under which the Chermak-Delgado lattice of a metabelian $p$-group of maximal class is a chain of length 0 . 
Corollary 4.2. Let $G$ be a metabelian p-group of maximal class. Then $\mathcal{C D}(G)$ is a chain of length 0 if and only if either $G$ possesses an abelian subgroup of index $p$ and $|G: Z(G)| \neq p^{2}$, or $G$ does not possess abelian subgroups of index $p,|G|>p^{5}$, and $|M: Z(M)|>p^{2}$ for any maximal subgroup $M$ of $G$.

We provide now a few remarks. The 2-groups of maximal class fall into 3 categories: the dihedral groups, the semidihedral groups, and the generalized quaternion groups (see e.g. Theorem 4.1 of [12], II), and each of these possess a cyclic subgroup of index 2 . Thus, the CD lattices of these groups fall under the umbrella of items 2 and 3 in Theorem 2.1.

Xue, Lv, and Chen provide sufficient conditions for when $p$-groups of maximal class have CD lattices that fall under the umbrella of item 3 in Theorem 2.1 (see Theorem 2.9 of [17]):

Proposition 4.3. Let $G$ be a finite $p$-group of order $p^{n}$ and of maximal class, where $p \geq 5$ and $n>2 p$. If $C_{G}(H)=Z(H)$ for every non-abelian subgroup $H$ of $G$, then $G$ possesses an abelian subgroup of index $p$.

Note that the finite non-abelian groups satisfying the condition in Proposition 4.3 are called CGZ-groups. Xue, Lv, and Chen show that if $G$ is a $p$-group of maximal class and $G$ is a CGZ-group, then $G$ is metabelian (see Proposition 2.6 of [17]).

We end with examples of metabelian $p$-groups of maximal class for each of the three cases in item 3 of Theorem 4.1. For the case a) such an example is presented in [17]:

$$
G=\left\langle a, b, c, d \mid a^{p^{2}}=b^{p}=c^{p}=d^{p}=1,[b, a]=c,[c, a]=d,[c, b]=[d, b]=a^{p},[d, a]=1\right\rangle,
$$

where $p \geq 5$.

For case b) we present $\operatorname{SmallGroup}\left(3^{6}, 99\right)$ from GAP:

$G=\langle a, b, c, d, e, f| a^{3}=e^{3}=f^{3}=1, b^{3}=c^{2} d, c^{3}=e^{2} f, d^{3}=f^{2},[b, a]=c,[c, a]=d,[d, a]=e$,

$[e, a]=[c, b]=f,[f, a]=[d, b]=[e, b]=[f, b]=[d, c]=[e, c]=[f, c]=[e, d]=[f, d]=[f, e]=1\rangle$. 
For case c) we present SmallGroup $\left(5^{6}, 651\right)$ from GAP:

$$
\begin{gathered}
G=\langle a, b, c, d, e, f| a^{5}=c^{5}=d^{5}=e^{5}=f^{5}=1, b^{5}=f^{3},[b, a]=c,[c, b]=d,[c, a]=[d, b]=e, \\
[d, a]=[e, b]=f,[e, a]=[f, a]=[f, b]=[d, c]=[e, c]=[f, c]=[e, d]=[f, d]=[f, e]=1\rangle .
\end{gathered}
$$

Acknowledgments. The authors are grateful to the reviewer for its remarks which improve the previous version of the paper.

\section{References}

[1] L. An, J.P. Brennan, H. Qu and E. Wilcox, Chermak-Delgado lattice extension theorems, Comm. Algebra 43 (2015), 2201-2213.

[2] B. Brewster and E. Wilcox, Some groups with computable ChermakDelgado lattices, Bull. Aus. Math. Soc. 86 (2012), 29-40.

[3] B. Brewster, P. Hauck and E. Wilcox, Groups whose Chermak-Delgado lattice is a chain, J. Group Theory 17 (2014), 253-279.

[4] B. Brewster, P. Hauck and E. Wilcox, Quasi-antichain ChermakDelgado lattices of finite groups, Archiv der Mathematik 103 (2014), 301-311.

[5] A. Chermak and A. Delgado, A measuring argument for finite groups, Proc. AMS 107 (1989), 907-914.

[6] G. Glauberman, Centrally large subgroups of finite p-groups, J. Algebra 300 (2006), 480-508.

[7] I.M. Isaacs, Finite group theory, Amer. Math. Soc., Providence, R.I., 2008.

[8] R. McCulloch, Chermak-Delgado simple groups, Comm. Algebra 45 (2017), 983-991.

[9] R. McCulloch, Finite groups with a trivial Chermak-Delgado subgroup, J. Group Theory 21 (2018), 449-461. 
[10] R. McCulloch and M. Tărnăuceanu, Two classes of finite groups whose Chermak-Delgado lattice is a chain of length zero, Comm. Algebra 46 (2018), 3092-3096.

[11] R. Schmidt, Subgroup lattices of groups, de Gruyter Expositions in Mathematics 14, de Gruyter, Berlin, 1994.

[12] M. Suzuki, Group theory, I, II, Springer Verlag, Berlin, 1982, 1986.

[13] M. Tărnăuceanu, The Chermak-Delgado lattice of ZM-groups, Results Math. 72 (2017), 1849-1855.

[14] L.S. Vieira, On p-adic fields and p-groups, Ph.D. Thesis, University of Kentucky, 2017.

[15] E. Wilcox, Exploring the Chermak-Delgado lattice, Math. Magazine 89 (2016), 38-44.

[16] M. Xu, L. An and Q. Zhang, Finite p-groups all of whose non-abelian proper subgroups are generated by two elements, J. Algebra 319 (2008), 3603-3620.

[17] H. Xue, H. Lv and G. Chen, On a special class of finite p-groups of maximal class, Italian J. Pure Appl. Math. 33 (2014), 279-284.

[18] A. Morresi Zuccari, V. Russo, and C.M. Scoppola, The ChermakDelgado measure in finite p-groups, J. Algebra 502 (2018), 262-276.

Ryan McCulloch

Assistant Professor of Mathematics

University of Bridgeport

Bridgeport, CT 06604

e-mail: rmccullo@bridgeport.edu
Marius Tărnăuceanu

Faculty of Mathematics

"Al.I. Cuza" University

Iaşi, Romania

e-mail: tarnauc@uaic.ro 